Original Article

\title{
Effects of active and passive light-touch support on postural stability during tandem standing
}

\author{
Miyoko Watanabe, RPT, PhD ${ }^{1 *}$, Hiroaki TAni, RPT, MD ${ }^{1)}$ \\ 1) International University of Health and Welfare: 2600-1 Kitakanemaru, Ohtawara City, \\ Tochigi Prefecture 324-8501, Japan
}

\begin{abstract}
Purpose] Light-touch support (consisting of a load $<100 \mathrm{~g}$ ) is useful for reducing postural sway while standing and walking. However, it is unclear which types of touch and somatosensory inputs are more effective for improving postural control. This study aimed to clarify the effects of active and passive light-touch support, with and without visual information, on postural stability during tandem standing. [Participants and Methods] Eleven young healthy adults maintained tandem standing for $30 \mathrm{~s}$ under six conditions. The independent variables were light-touch condition (no, active or passive) and visual condition (eyes open or closed). Postural sway, as measured using a force plate, was considered to indicate postural stability. [Results] There was significantly less postural sway with active light touch compared with passive and no touch. Passive light touch resulted in significantly less sway than no touch. In addition, there was less postural sway with active light compared to passive light touch, especially with the eyes closed. The light-touch support force did not differ between conditions. [Conclusion] Both active and passive light-touch improved postural stability in comparison to no-touch. Active light-touch support significantly improved postural stability. These results could be used to inform therapeutic interventions within clinical settings. Key words: Light touch, Activity, Postural stability
\end{abstract}

(This article was submitted Aug. 22, 2019, and was accepted Oct. 17, 2019)

\section{INTRODUCTION}

Light-touch support is used in index finger contact with external objects, with a force of less than $100 \mathrm{~g}$ (1 N). Previous research showed that only light-touch support with fingertip contact, not sufficient to support body weight, generally enhanced postural stability ${ }^{1-3)}$. Moreover, the effects of light-touch support on postural stability appear especially dependent on visual information ${ }^{3,4)}$. The effects of light-touch support on postural stability are seen in elderly individuals ${ }^{5)}$, individuals with hemiparesis ${ }^{6}$, and those with Parkinson's disease ${ }^{7}$. Light-touch support therefore improved postural stability in both healthy and patient populations ${ }^{8}$.

One factor explaining the effect of light-touch support is that somatosensory information is important for postural stability. With light-touch support during standing, somatosensory information input is transmitted to the body by the finger and the information can facilitate postural stability. Previous research ${ }^{9)}$ reported that individuals with profound sensory neuropathy could not obtain the benefit of light-touch support for postural stability because they could not receive somatosensory information. Another research ${ }^{10}$ also reported that the effects of light-touch support could not be shown for participants with sensory input blocked by tourniquet ischemia. Somatosensory information during postural control enhances stability, but it is unclear how effective somatosensory information is processed by the body.

In a clinical setting, physical therapists often touch a patient's trunk, back, or shoulder during standing or walking to prevent postural instability and falls. This manual light touch is not intended to support the patient's body weight. Can passive light touch by another individual, i.e., passive light touch, also enhance postural stability? If somatosensory information input

*Corresponding author. Miyoko Watanabe (E-mail: mwatanabe@iuhw.ac.jp)

(C2020 The Society of Physical Therapy Science. Published by IPEC Inc.

(c) (1) $($ This is an open-access article distributed under the terms of the Creative Commons Attribution Non-Commercial No Derivatives

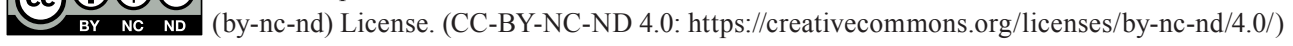


is important for stable posture, passive light touch by others might be as effective for postural stability as active light touch, because the somatosensory information is the same as that provided by self-input light touch.

This study aimed to clarify the effect of light-touch support on postural stability. We compared the effects of active and passive light-touch support, with and without visual information, on postural stability during tandem standing. We hypothesized that both passive and active light touch could enhance postural stability by a similar mechanism, and that the effects of passive/active light-touch on postural stability would be stronger in the absence of visual information.

\section{PARTICIPANTS AND METHODS}

Eleven (6 males and 5 females) right-handed young adults with mean age 22.1 years \pm 2.8 , height $166.1 \mathrm{~cm}$ \pm 5.5 , and weight $58.5 \mathrm{~kg} \pm 7.9$ participated in this study. None had a history of orthopedic injury or paretic disorder. All participants provided informed consent in written and verbal prior to the study. The local ethics committee approved the research (number 10-214). All procedures used in this study were in accordance with the Helsinki Declaration.

Participants stood on a force plate with the right (that is dominant side) heel and left (that is non-dominant side) toe in contact with each other, with the feet in a straight line, i.e., tandem standing. Participants were asked to stand for $30 \mathrm{~s}$ without postural sway under 6 conditions using 2 variables: light touch condition (no, active or passive) and visual condition (eyes open or closed). Participants performed the task 2 times under each condition. The 6 experimental conditions were counterbalanced with a presentation order using a Latin square design.

Center of pressure (COP) displacement as postural sway was measured on a force plate (Twin Gravicorder GP-6000; Anima) that collected data for $30 \mathrm{~s}$ with a sampling rate set at $50 \mathrm{~Hz}$. The force of light-touch support during the task was measured as the vertical force on a load cell (LUR-A-1KNSA1; Kyowa). The load cell was placed on a table under active conditions for contact by the participant. Analog output from the load cell was processed through an amplifier (YB-530; Kyowa), converted into digital data at a sampling rate of $200 \mathrm{~Hz}$, and stored in a data collection system (NR-2000; Keyence).

The participants were asked to perform tandem standing and to sway as little as possible for $30 \mathrm{~s}$. The arms were relaxed at the side during all tasks. For the active and passive light-touch condition, participants touched the load cell under $100 \mathrm{~g}$ force using the index fingertip ${ }^{1-3)}$. For the active condition, participants performed light touch using self-adjustment of force. The load cell was placed on a table at wrist height. For the passive condition, participants were asked to make fingertip contact with the load cell that was held by researcher. The researcher held the load cell at the participant's wrist height and touched the participant's index finger with no more than $100 \mathrm{~g}$ of force (Fig. 1). The researcher maintained the force of light-touch support, with confirmation using a digital oscilloscope monitor (TDS-2014; Tektronix). The monitor showed the force on the load cell. Therefore, if the participant changed position and the light-touch support increased to more than $100 \mathrm{~g}$, the researcher decreased the force of light touch support. Before the experimental trial, participants and researchers practiced applying light-touch support of no more than $100 \mathrm{~g}$. If the participant supported over $100 \mathrm{~g}$ in the experimental trial, the participant redid the trial. For the no-touch condition, participants stood without upper limb support.

With regard to the visual condition, the participants gazed straight ahead at a fixation target point on a wall $1.5 \mathrm{~m}$ away in the eyes open condition, and closed their eyes with the head pointed toward the fixation target point in the eyes closed condition.

Mean values of two trials for each of the dependent variables (i.e., the path length of the COP and the force of lighttouch support) were calculated. The path length of the COP for each condition was transformed to Z-scores (mean $[\mathrm{M}]=50$, standard deviation $[\mathrm{SD}]=10$ ) to eliminate the effect of individual bias. Two-way analysis of variance (ANOVA) was used for the Z-scores of COP path length, and the mean light-touch support force was analyzed with two-factor repeated measures. For all analyses, a p-value less than 0.05 was considered significant.

\section{RESULTS}

ANOVA for the mean Z-scores of COP path length (Table 1) showed a significant main effect for the light-touch condition $(\mathrm{F} 2,20=75.3, \mathrm{p}<0.05)$, with the values being significantly lower for the active light-touch condition (M 43.9, SD 3.8) than for 
Table 1. The mean Z-scores of COP path length under both light-touch and visual conditions

\begin{tabular}{lllcc}
\hline & & \multicolumn{3}{c}{ Light-touch condition } \\
\cline { 3 - 4 } & & No-touch & Passive & Active \\
\hline Visual condition & Eyes open & $49.5 \pm 8.3^{*}$ & $43.0 \pm 4.0^{* \dagger}$ & $40.9 \pm 3.3^{* \dagger}$ \\
& Eyes closed & $66.4 \pm 5.8$ & $53.3 \pm 5.6^{\dagger}$ & $46.8 \pm 4.4^{\dagger \S}$ \\
\hline
\end{tabular}

*: vs. Eyes closed condition, $\uparrow$ : vs. No-touch condition, $\S$ : vs. Passive condition.

Table 2. The force of support under light-touch and visual conditions

\begin{tabular}{llll}
\hline & & \multicolumn{2}{c}{ Light-touch condition } \\
\cline { 3 - 4 } & & Passive $(\mathrm{g})$ & Active $(\mathrm{g})$ \\
\hline Visual condition & Eyes open & $64.7 \pm 24.6$ & $60.6 \pm 33.8$ \\
& Eyes closed & $62.5 \pm 28.3$ & $71.1 \pm 33.3$ \\
\hline
\end{tabular}

the passive (M 48.2, SD 4.8) and no-touch condition (M 57.9, SD 7.0); the values were also significantly lower for the passive light-touch condition than for the no-touch condition $(\mathrm{p}<0.05)$. The mean Z-score for the COP path length was significantly lower for the passive light-touch condition than for the no-touch condition $(\mathrm{p}<0.05)$.

The main effect for the visual condition was also significant (F1, 10=18.6, p $<0.05$ ), with the mean Z-scores of the COP path length being significantly lower in the eyes open condition (M 44.5, SD 5.2) than in the eyes closed condition (M 55.5, SD 5.2).

The interaction between the two factors (the light-touch condition and the visual condition) was significant $(F 2,20=5.7$, $\mathrm{p}<0.05$ ). Subsequent simple main effect analysis indicated that the mean Z-score of the COP path length in the active lighttouch condition (M 46.8, SD 4.4) was significantly lower than in the passive light-touch condition (M 53.3, SD 5.6) and no-touch condition (M 66.4, SD 5.8) with the eyes closed ( $\mathrm{p}<0.05)$. In contrast, the mean Z-score of the COP path length in the active light-touch condition (M 40.9, SD 3.3) was significantly lower than in the no-touch (M 49.5, SD 8.3) condition with the eyes open $(\mathrm{p}<0.05)$. However, the mean Z-scores of the COP path length in the active light-touch (M 40.9, SD 3.3) and passive light-touch condition (M 43.0, SD 4.0) were not significantly different.

For the force of light-touch support (Table 2), two-way ANOVA revealed no significant main effect for the light-touch condition $(\mathrm{F}<1)$ and the visual condition $(\mathrm{F}<1)$. The interaction between the two factors was also not significant $(\mathrm{F}<1)$.

\section{DISCUSSION}

Postural stability in both the active and passive light-touch conditions was better than in the no-touch condition. This finding was consistent with previous reports ${ }^{1-3)}$. The effectiveness of both active and passive light-touch support could be useful in clinical settings. Patients with unstable posture during standing or gait may require only light support by the therapist to maintain posture instead of firm or self-support.

Results of this study showed that postural stability in the active light-touch support condition was better than in the passive light-touch support condition. The active light-touch condition was performed using self- input of somatosensory information from the fingertip. Although light touch provided very little support, self-performed light touch was effective for postural stability. Therefore, active input of somatosensory information contributed to postural stability.

The effect of active light touch on postural stability was especially apparent with the eyes closed. This suggested that active input of somatosensory information was important in the absence of visual information. Dickstein et al. ${ }^{11}$ examined the effects of light-touch contact during walking, with and without visual information, and observed that in the absence of light touch and visual information (i.e., eyes closed), the center of mass shifted backward and caused postural instability; however, the postural instability improved with light-touch contact. Their study suggested that light touch provided spatial orientation of body, which had effectiveness similar to that of vision. Jeka et al. ${ }^{1)}$ also examined the effects of light-touch contact during tandem standing, with and without visual information, and found that light-touch contact was more effective than vision in reducing postural sway. Maeda et al. ${ }^{12}$ compared postural stability using cane support and light contact with a wall in elderly individuals with visual impairment, and found that postural sway with wall contact was less than that with cane support. Therefore, active light touch provided significant information about spatial orientation and maintained body stability during tandem standing, especially in the absence of visual information.

Results according to the force of light-touch support showed no differences under the 6 conditions. The force of lighttouch support was not affected by how the support was applied, regardless of visual input. Therefore, differences in postural stability under the 6 conditions were not related to the force of light-touch support. Somatosensory input rather than the force of light-touch support was necessary for postural stability.

The findings of this study can be applied in a clinical rehabilitation setting. If an apprehensive patient relies on upper limb support using parallel bars, crutches, or assistive devices despite the ability to stand and walk without support, a therapist 
should first ask the patient to apply light touch, less than that required for full support. This could stabilize posture, because light-touch support is more effective than no touch. Moreover, active light touch is more effective than passive light touch for postural stability. A patient with instability should lightly touch an external object. Active light-touch support is more effective with eyes closed. Patients should use active light-touch support in the absence of vision or when in the dark.

A limitation of this study was that the mechanism by which active light touch without visual input was effective for postural stability was not clear. Even though the force of light-touch support was very small, this light contact contributed to stable posture. Future research should clarify the applications for active light-touch support.

\section{Funding}

This work was supported by JSPS KAKENHI Grant Number JP17K18053.

\section{Conflict of interest}

There are no conflicts of interest.

\section{REFERENCES}

1) Jeka JJ, Lackner JR: Fingertip contact influences human postural control. Exp Brain Res, 1994, 100: 495-502. [Medline] [CrossRef]

2) Jeka JJ: Light touch contact as a balance aid. Phys Ther, 1997, 77: 476-487. [Medline] [CrossRef]

3) Holden M, Ventura J, Lackner JR: Stabilization of posture by precision contact of the index finger. J Vestib Res, 1994, 4: 285-301. [Medline]

4) Forero J, Misiaszek JE: The contribution of light touch sensory cues to corrective reactions during treadmill locomotion. Exp Brain Res, 2013 , 226: 575-584. [Medline] [CrossRef]

5) Baccini M, Rinaldi LA, Federighi G, et al.: Effectiveness of fingertip light contact in reducing postural sway in older people. Age Ageing, 2007, 36: 30-35. [Medline] [CrossRef]

6) Cunha BP, Alouche SR, Araujo IM, et al.: Individuals with post-stroke hemiparesis are able to use additional sensory information to reduce postural sway. Neurosci Lett, 2012, 513: 6-11. [Medline] [CrossRef]

7) Franzén E, Paquette C, Gurfinkel V, et al.: Light and heavy touch reduces postural sway and modifies axial tone in Parkinson's disease. Neurorehabil Neural Repair, 2012, 26: 1007-1014. [Medline] [CrossRef]

8) Baldan AM, Alouche SR, Araujo IM, et al.: Effect of light touch on postural sway in individuals with balance problems: a systematic review. Gait Posture, 2014, 40: 1-10. [Medline] [CrossRef]

9) Dickstein R, Peterka RJ, Horak FB: Effects of light fingertip touch on postural responses in subjects with diabetic neuropathy. J Neurol Neurosurg Psychiatry, 2003, 74: 620-626. [Medline] [CrossRef]

10) Kouzaki M, Masani K: Reduced postural sway during quiet standing by light touch is due to finger tactile feedback but not mechanical support. Exp Brain Res, 2008, 188: 153-158. [Medline] [CrossRef]

11) Dickstein R, Laufer Y: Light touch and center of mass stability during treadmill locomotion. Gait Posture, 2004, 20: 41-47. [Medline] [CrossRef]

12) Maeda A, Nakamura K, Otomo A, et al.: Body support effect on standing balance in the visually impaired elderly. Arch Phys Med Rehabil, $1998,79: 994-997$. [Medline] [CrossRef] 